\title{
ARTICLE
}

\section{Study of angular and energy distribution of secondary electrons emitted by high energy heavy ion impact}

\author{
Katashi KIYOHARA $^{1 *}$, Yusuke KOBA ${ }^{1}$, Hiroki IWAMOTO ${ }^{1}$, Toshinori NAGASAKI ${ }^{1}$ \\ and Yusuke UOZUMI ${ }^{1}$ \\ ${ }^{1}$ Kyushu University, 744, Motooka, Nishi-ku, Fukuoka 819-0395, Japan
}

\begin{abstract}
To study the ion track structure in an inorganic scintillator with the Monte Carlo method, ionization cross sections for angular- and energy-distributions were extended from the available data. Some typical behaviors of secondary electrons were calculated in terms of the heavy-ion interactions with water at energies from $1 \mathrm{MeV}$ to $1 \mathrm{GeV}$. Their energy distributions were compared between different ions and energies.
\end{abstract}

\section{KEYWORDS: ion track structure, inorganic scintillator, ionization cross section, secondary electrons}

\section{Introduction}

The scintillation efficiency of inorganic crystals has been long-time controversial ${ }^{1)}$ in terms of energetic heavy-ion bombardment. The responses of crystalline scintillators were studied intensively, and experimental results have previously shown that the scintillation efficiency of crystals to heavy-ions decreases with increasing specific energy loss of ions. Moreover, it is not a function of $d E / d x$ alone, but is instead composed of a series of discrete function, on for each incident ions. We carried out light output measurements at HIMAC of the National Institute for Radiological Sciences. The results show for instance, the scintillation efficiency of ${ }^{40} \mathrm{Ar}$ ions is about twice that of ${ }^{12} \mathrm{C}$ ions at a stopping power of around $10^{3} \mathrm{MeVcm}^{2} / \mathrm{g}$.

This ion-identity dependence has been explained rather qualitatively with the track structure model ${ }^{2}$. However, there have been no quantitative analyses conducted so far. In order to obtain the deep understanding of the track structure, which might govern the ion-identity dependence, the development of a Monte Carlo simulation code is crucial. Since the cross section data is very scarce and limited to proton-water interactions at low energies below a few $\mathrm{MeV}$, it is needed to estimate cross sections.

In the present study, we extend the data of proton-water interactions to the higher energy range up to $1 \mathrm{GeV}$. Additionally, the extension to heavy-ion incidence ionization cross sections is made from the data of proton incidence one. Using these results, we conduct calculations of secondary electron behaviors in water to discuss the basic nature of the ion track structure.

\footnotetext{
*Corresponding Author,

phone No. +81-92-802-3479

fax no. . +81-92-802-3479

E-mail:katakata@nucl.kyushu-u.ac.jp
}

\section{Calculation Method}

1. Ionization cross section

The ionization cross sections for the particle impact on molecule were calculated by referring to the electron impact ionization cross section. Following an energy scaling ${ }^{3)}$, the ionic particle cross section at a kinetic energy is given by the electron cross section at the energy, where the velocity is the same as that of the ion. In the relativistic kinematics, the relation between the kinetic energy $T$ and the velocity $v$ is given by

$$
T=\left\{\frac{1}{\sqrt{1-(v / c)^{2}}}-1\right\} m_{0} c^{2} .
$$

The prescription formulated by Seltzer ${ }^{4)}$ gives electron ionization cross sections on a water molecule. The energy-differential cross sections in terms of electron kinetic energy $\varepsilon$ ejected from the $j$-th orbital of the molecule are written in two terms,

$$
\frac{d \sigma^{(j)}}{d \varepsilon}=\frac{d \sigma_{c}{ }^{(j)}}{d \varepsilon}+\frac{d \sigma_{d}{ }^{(j)}}{d \varepsilon} .
$$

The first term is the close collision between two electrons, and expressed in the form:

$$
\begin{gathered}
\frac{d \sigma_{c}^{(j)}}{d \varepsilon}=\frac{2 \pi r_{e}{ }^{2} m_{e} c^{2} n_{j}}{\eta^{2}} \frac{T}{T+B_{j}+U_{j}} \cdot \\
\left\{\frac{1}{E^{2}}+\frac{1}{(T-\varepsilon)^{2}}+\frac{1}{T^{2}}\left(\frac{\tau}{\tau+1}\right)^{2}\right\} \\
\left\{-\frac{1}{E(T-\varepsilon)} \frac{2 \tau+1}{(\tau+1)^{2}}+G_{j}\right\}
\end{gathered}
$$

with

$$
\left.G_{j}=\frac{8 U_{j}}{3 \pi}\left\{\frac{1}{E^{2}}+\frac{1}{(T-\varepsilon)^{3}}\right\} \tan ^{-1} \sqrt{\xi}+\frac{\sqrt{\xi}(\xi-1)}{(\xi+1)^{2}}\right\}
$$

and

$$
\begin{aligned}
& \eta^{2}=1-(\tau+1)^{-1}, \\
& \tau=T / m_{e} c^{2}, \\
& y=\varepsilon / U_{j} .
\end{aligned}
$$


Here we used $r_{e}=2.81794 \times 10^{-13} \mathrm{~cm}$, and $m_{e} c^{2}$ is the electron rest mass, $n_{j}$ the number of electrons in the orbital, $B_{j}$ the orbital binding energy, $U_{j}$ the mean kinetic energy of the orbital electron, and $T$ the kinetic energy of incident electron. The energy transfer $E$ is given by $E=\varepsilon+B_{j}$

The second term comes from the interaction with the equivalent radiation field, and is written by

$$
\frac{d \sigma_{d}{ }^{(j)}}{d \varepsilon}=n_{j} I(E) \sigma_{P E}^{(j)}(E),
$$

where $\sigma_{P E}^{(j)}$ is the photoelectric cross sections per orbital electron for the incident photon of energy $E\left(=\varepsilon+B_{j}\right)$. The virtual photon spectrum integrated over impact parameters $b_{\text {min }}<b<b_{\text {max }}$ is given by

$$
I(E)=\frac{2}{137 \pi \beta^{2} E}\left\{H\left(x_{\min }\right)-H\left(x_{\max }\right)\right\},
$$

with

$$
H(x)=x K_{0}(x) K_{1}(x)-\frac{x^{2}}{2}\left\{K_{1}^{2}(x)-K_{0}^{2}(x)\right\},
$$

where

$$
x=\frac{E b}{\hbar} \frac{\sqrt{1-\eta^{2}}}{\eta},
$$

$K_{0}$ and $K_{1}$ are the Bessel function of the order of 0 and 1 , respectively.

The partial ionization cross section for the molecular orbit $j$ is written by

$$
\sigma^{(j)}=\int_{0}^{\left(T-B_{j}\right) / 2} d \varepsilon \frac{d \sigma^{(j)}}{d \varepsilon}
$$

The total ionization cross section is given by

$$
\sigma_{\text {ion }}=\sum_{j=1}^{5} \sigma^{(j)}
$$

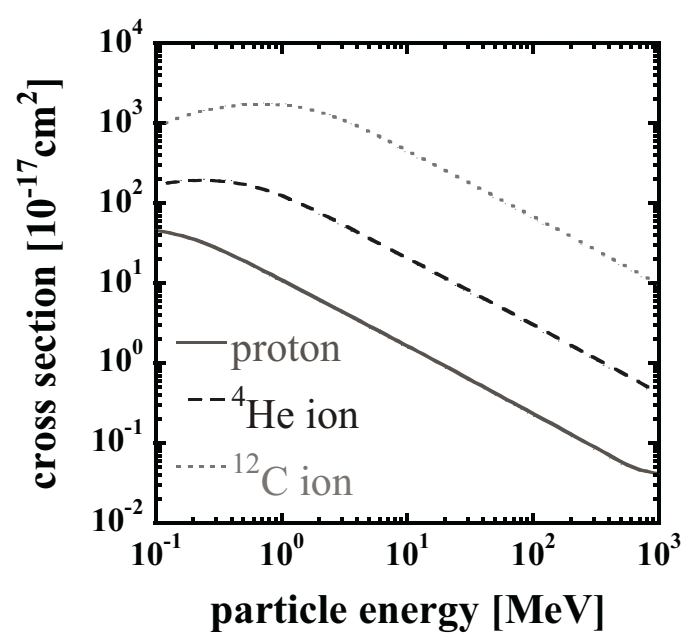

Fig.1 Ionization cross section of water molecule.
As an example of the formalism, the ionization cross sections for water vapor were calculated for proton, ${ }^{4} \mathrm{He}$ and ${ }^{12} \mathrm{C}$ ion bombardment in an energy range from 0.1 to 1000 $\mathrm{MeV}$. Resultant cross sections are shown in Fig. 1 for ${ }^{4} \mathrm{He}-$ and ${ }^{12} \mathrm{C}$-interactions with $\mathrm{H}_{2} \mathrm{O}$ molecule as well as proton- $\mathrm{H}_{2} \mathrm{O}$ interactions.

\section{Energy transfer}

The energy transfer in a single collision was calculated so as to be realistic. First, the flight distance before a collision $d s$ is determined by

$$
d s=\frac{\log (r)}{\sigma_{\text {ion }} \rho_{e}},
$$

where $r$ is random parameter, $\sigma_{i o n}$ and $\rho_{e}$ are the ionization cross section and the electron number density of the medium.

Secondly, the mean energy loss $\Delta E_{\text {mean }}$ after a step $d s$ is computed by

$$
\Delta \mathrm{E}_{\text {mean }}=\frac{d E}{d x} \cdot d s,
$$

with so-called Bethe-Bloch formula

$$
\begin{aligned}
\frac{d E}{d x} & =4 \pi r_{e}{ }^{2} z^{2} \frac{m_{e} c^{2}}{\beta^{2}} N Z \\
& \cdot\left\{\log \left(\frac{2 m_{e} c^{2}}{I} \beta^{2}\right)-\log \left(1-\beta^{2}\right)-\beta^{2}\right\},
\end{aligned}
$$

where $z$ is atomic number of incident particle, $m_{e} c^{2}$ is rest mass energy of electron, $\beta$ is light speed ratio, $N$ and $Z$ are number density and atomic number of medium and $I$ is mean excitation energy.

Finally, the actual energy is determined by taking the energy straggling into consideration. The formulae used to compute the energy straggling in a thin layer of matter are first described theoretically by Landau. They give rise to a universal asymmetric probability density function characterized by a narrow peak with a long tail towards positive values due to the small number of individual collisions, each with a small probability of transferring comparatively large amounts of energy. The mathematical definition of the probability density function ${ }^{5)}$ is

$$
\varphi(\lambda)=\frac{1}{2 i \pi} \int_{c-i \infty}^{c+i \infty} e^{x \log (x)+\lambda x} d x
$$

where $\lambda$ is a dimensionless number and $c$ is proportional to the energy loss, and $\mathrm{c}$ is any real positive number. The best existing fitting function is probably the so-called Moyal function as presented by Rotondi and Montagna ${ }^{6}$. However, this function is unsuitable for rapid sampling methods. In the present work, the process computes the actual energy loss $\Delta E$ by using a closed analytic form ${ }^{7}$

$$
\Psi(\lambda)=\sqrt{\frac{e^{\left(\lambda+e^{\lambda}\right)}}{2 \pi}}
$$

with

$$
\lambda=\frac{\Delta E-\Delta E_{\text {mean }}}{\xi},
$$




$$
\xi=4 \pi r_{e}^{2} z^{2} \frac{m_{e} c^{2}}{\beta^{2}} N Z \cdot d s
$$

\section{Scattering angle}

The scattering angles $\theta$ in the centre-of-mass frame of the incident particle and the secondary electron is determined by using Rutherford formula ${ }^{8)}$

$$
\frac{E_{1}-\Delta E}{E_{1}}=\frac{m_{1}^{2}+m_{2}^{2}+2 m_{1} m_{2} \cos \theta}{\left(m_{1}+m_{2}\right)^{2}},
$$

where $E_{1}$ and $m_{1}$ are kinetic energy and rest mass of the incident particle, $m_{2}$ is rest mass of electrons. The scattering angle of the incident particle $\theta_{1}$ and that of secondary electron $\theta_{2}$ in the laboratory frame $\left(\theta_{1}\right.$ and $\theta_{2}$ are defined in Fig. 2.) is given with $\theta$ :

$$
\theta_{1}=\arctan \frac{\sin \theta}{m_{1} / m_{2}+\cos \theta}
$$

and

$$
\theta_{2}=\frac{1}{2}(\pi+\theta)
$$

Therefore, the scattering angles $\theta_{1}$ and $\theta_{2}$ are determined by (14), (15) and (16) with $E_{1}$ and $\Delta E$.

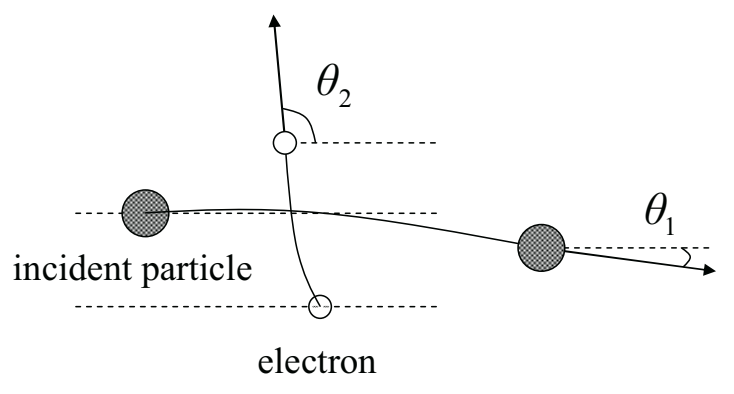

Fig. 2 Scattering angle definition.

\section{Results}

\section{Tracking and the scattering angles of electrons}

The code simulates the scattering of particle in the water. An example of incident particle $\left({ }^{12} \mathrm{C}\right.$ ion) tracking, which is shown in Fig. 3, is reproduced by the code. Hence we are planning to investigate the straggling of angle which is perpendicular to incident direction by using the code.

The code also simulated the scattering angle of electron. The mean scattering angle of an electron recoiled by the incident ion is shown in Fig. 4. It is found that the scattering angle concentrates to around 90-degree. However, higher incident particle energy is, since shorter the interaction time is, smaller the mean of electron scattering angle is. Moreover, the mean and standard deviation of the scattering angles in the region of low incident particle energy depend on atomic number of the incident particle. The scattering angle mean of secondary electrons for ${ }^{12} \mathrm{C}$ ion is the smallest and that for proton is the largest.

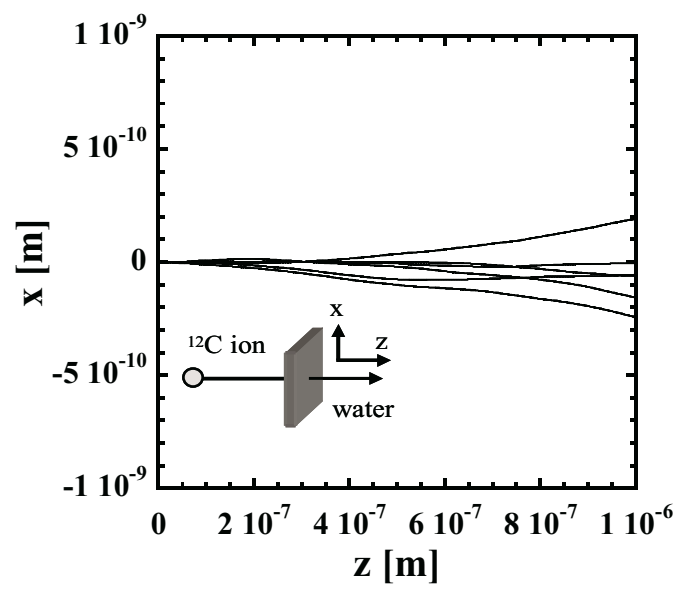

Fig. 3 An example of the incident particle tracking.

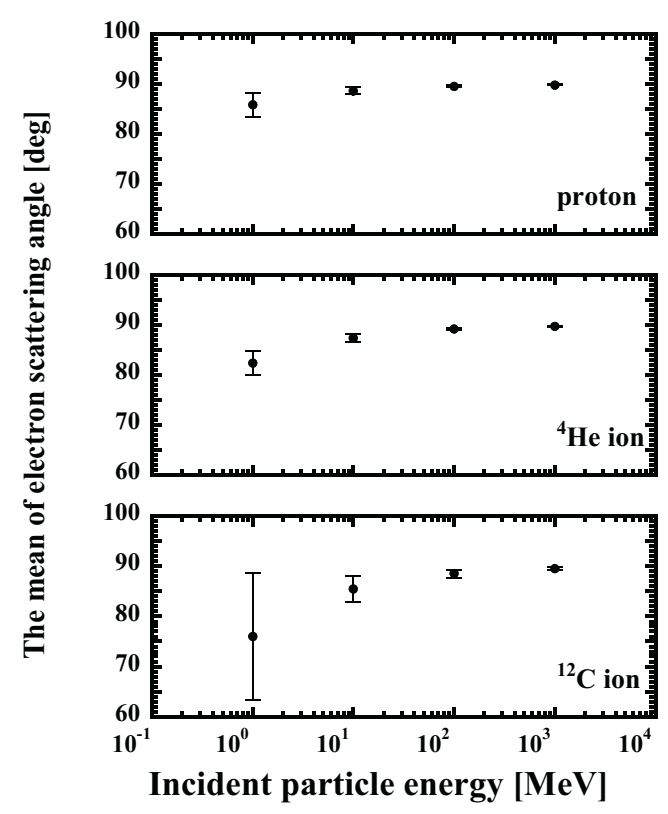

Fig. 4 The scattering angles of secondary electrons.

\section{Energy distribution of electrons}

In Fig. 5 are displayed energy distributions of secondary electrons from a water slab of $1 \mathrm{~nm}$ thick bombarded by protons, ${ }^{4} \mathrm{He}$ and ${ }^{12} \mathrm{C}$ ions at various energies. It is appeared that the number of low energy electrons increases significantly with increasing the atomic number. In the case of the ${ }^{12} \mathrm{C}$-ion incidence, the increase of low-energy part is remarkable with decreasing the kinetic energy.

In Fig. 6, energy distributions are compared between protons, ${ }^{4} \mathrm{He}$ and ${ }^{12} \mathrm{C}$ ions at the same speed. Due to the difference in cross sections, the distributions look different as shown in the left panel. As in the right panel, however, the distribution shapes are almost same. As well, the vertical axis of the right panel is normalized counts. These values are divided by each count of which electron energy is $1 \mathrm{eV}$. This result implies that the energy distribution of secondary electrons depends on the speed of incident particles. 

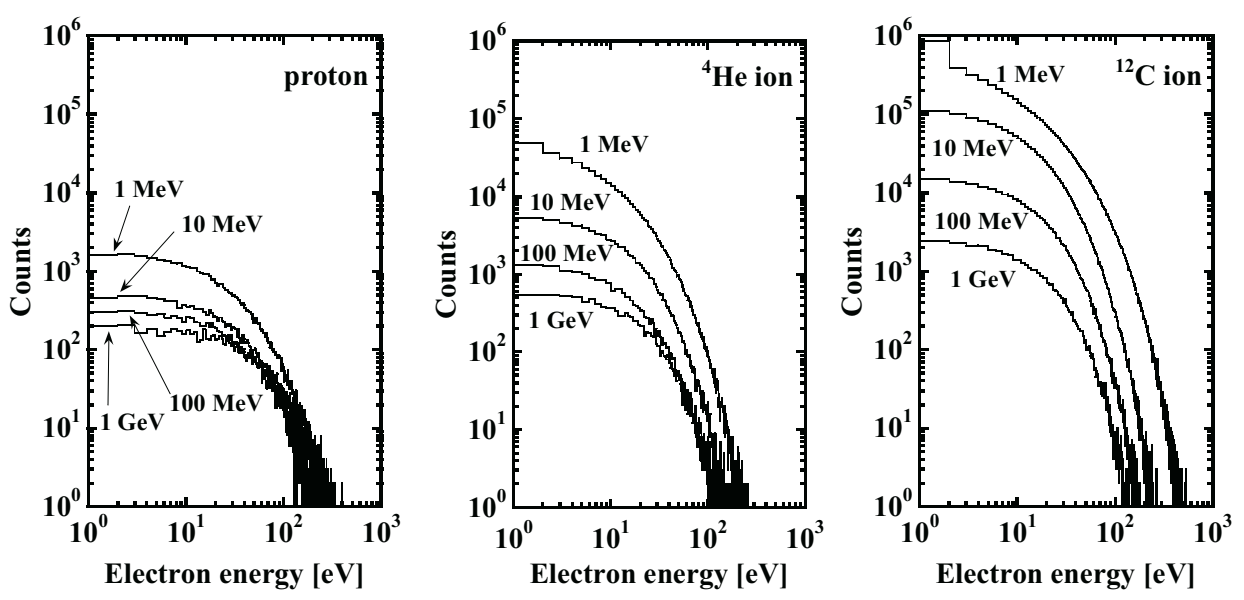

Fig. 5 Energy distribution of electrons from impact of proton, ${ }^{4} \mathrm{He}$ and ${ }^{12} \mathrm{C}$ ions.

From the above results, a qualitative explanation for ion track structure can be extracted: the significant increase of low-energy electrons leads to a generation of high-density region where the scintillation efficiency is suppressed due to the high-temperature. The ion-identification dependence is possibly attributable to the density of secondary electrons rather than the spatial distribution that is determined by the electron energy distribution.

\section{Conclusion}

In order to interpret the ion-identity dependence of the crystalline scintillator response, we investigated elemental physical quantities which might govern the formation of the ion track and conducted a theoretical study by the Monte Carlo simulation. We choose water in place of crystalline scintillators because of many cross section data available.

By using the simulation code developed presently, we investigated the angle and the energy distribution of secondary electrons. Simulation results have suggested the angle distribution and the number of low-energy electrons depend on the atomic number of incident particle and the energy distribution shapes of secondary electrons depend on the speed of incident particle.

In near future, we will set inorganic scintillators as the target to investigate the relation between the scintillators response and the ion track structure. Though the present result has provided us an intuitive understanding of ion track structure, the qualitative interpretation will be obtained in our future work with extending the cross section data from water to crystal material.

\section{References}

1) M. Pârlog et al., "Response of CsI(Tl) scintillators over a large range in energy and atomic number of ions", Nucl. Instrum. Methods A, 482, 674 (2002), and references therein.
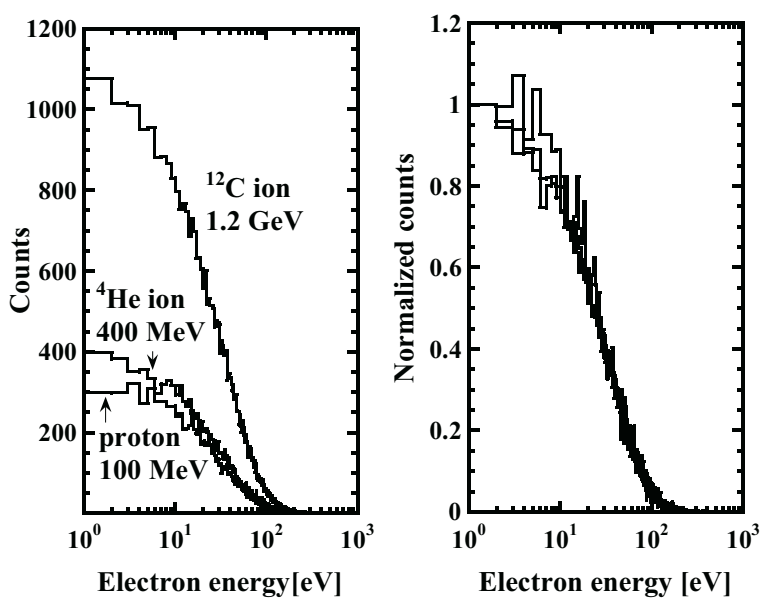

Fig. 6 Electron energy distribution for the same speed incident particles of proton, ${ }^{4} \mathrm{He}$ and ${ }^{12} \mathrm{C}$ ions. The right is the same as the left, but normalized peak values to unity.

2) M. Luntz, "Track-effect theory of scintillation efficiency", Phys. Rev. B, 4,2857 (1971).

3) H. Nikjoo, S. Uehara, D. Emfietzoglou and A. Brahme, "Heavy charged particles in radiation biology and biophysics", J. Phys., 10, 1 (2008).

4) S. M. Seltzer, "Cross sections for bremsstrahlung production and electron-impact ionization, Monte Carlo Transport of electrons and photons edited by T. M. Jenkins and A. Rindi", New York Plenum, 81 (1988).

5) L. Landau, J. Phys. (USSR), 8, 204 (1944).

6) A. Rotondi, and P. Montagna, "Fast calculation of Vavilov distribution.”, Nucl. Instrum. Methods B, 47, 215 (1990).

7) J. E. Moyal, "Theory of ionization fluctuations", Phil. Mag., 46, 263 (1955).

8) C. Lehmann, "Interaction of Radiation with Solids and Elementary Defect Production", Elsevier North-Holland, New York, 3 (1978). 